\title{
The Navier-Stokes equations and backward uniqueness
}

\author{
G. Seregin \& V. Šverák \\ Dedicated to Olga Alexandrovna Ladyzhenskaya
}

Abstract We consider the open problem of regularity for $L_{3, \infty}$-solutions to
the Navier-Stokes equations. We show that the problem can be reduced to a
backward uniqueness problem for the heat operator with lower order terms.

1991 Mathematical subject classification (Amer. Math. Soc.): 35K, $76 \mathrm{D}$.

Key Words: the Navier-Stokes equations, the Cauchy problem, weak LerayHopf solutions, suitable weak solutions, backward uniqueness.

\section{Introduction}

In this paper, we deal with the classical Cauchy problem for the Navier-Stokes equations:

$$
\begin{gathered}
\partial_{t} v(x, t)+\operatorname{div} v(x, t) \otimes v(x, t)-\Delta v(x, t)=-\nabla p(x, t), \\
\operatorname{div} v(x, t)=0
\end{gathered}
$$

for $x \in \mathbb{R}^{3}$ and for $t>-T_{0}$, and

$$
v\left(x,-T_{0}\right)=a(x), \quad x \in \mathbb{R}^{3} .
$$

Problem (1.1), (1.2) has at least one weak solution $v$ in the so-called Leray-Hopf class, see [8] and [4].

It is known (see [2], [5]-[7], [9], [12], [15], [18], and [19]) that, under the additional condition

$$
v \in L_{s, l}\left(-T_{0}, T ; \mathbb{R}^{3}\right), \quad \frac{3}{s}+\frac{2}{l} \leq 1, \quad s \geq 3, \quad l \geq 2,
$$


the weak Leray-Hopf solution is unique on the interval $]-T_{0}, T[$. Moreover, this solution is smooth if $s>3$. It is an open problem whether weak solutions remain to be smooth if $s=3$ and $l=+\infty$. See [13],[14], and [16] for various results related to this problem.

In this note, we connect the above problem to a backward uniqueness problem for the heat equation. The problem seems to be of independent interest from the point of view of control theory.

We outline the main idea. Assume that $\left.(0,0) \in \mathbb{R}^{3} \times\right]-T_{0},+\infty[$ is a singular point of a solution $v$ satisfying

$$
\text { ess } \sup _{-T_{0}<t<+\infty} \int_{\mathbb{R}^{3}}|v(x, t)|^{3} d x<+\infty .
$$

For $\lambda>0$, we consider functions

$$
v_{\lambda}(x, t)=\lambda v\left(\lambda x, \lambda^{2} t\right)
$$

defined in $\left.\mathbb{R}^{3} \times\right]-T_{0} / \lambda,+\infty[$. The crucial point is that both equations (1.1) and condition (1.3) ( with $T_{0}$ replaced by $T_{0} / \lambda$ ) are invariant under scaling (1.4). Moreover, compactness properties of weak solution enable us to pass to the limit as $\lambda \rightarrow 0+0$ along a suitable subsequence $\lambda_{j} \rightarrow 0+0$

The result of this procedure is a solution $u=\lim v_{\lambda_{j}}$ to the Navier-Stokes equations which is non-trivial (unless $(0,0)$ is a regular point of $v$ ), is defined on $\mathbb{R}^{3} \times \mathbb{R}$, and vanishes for $t>0$. Moreover, $u$ is regular in space-time domains of the form

$$
\left.\left\{\mathbb{R}^{3}-B(0, R)\right\} \times\right]-T_{1},+\infty[
$$

where $R=R\left(T_{1}\right)$. We now consider the equation for the vorticity $\omega=\nabla \wedge u$ which is

$$
\partial_{t} \omega+\omega_{, k} u_{k}-u_{, k} \omega_{k}-\Delta \omega=0 .
$$

We view (1.5) as a linear heat equation for $\omega$ with lower order terms

$$
\partial_{t} \omega-\Delta \omega=A_{k} \omega_{, k}+B \omega
$$

where $A=\left(A_{k}\right)$ and $B=\left(B_{i j}\right)$ are given functions.

We now conjecture the following.

Conjecture. Assume that $A$ and $B$ have reasonable regularity properties and suitable decay at $\infty$. Assume that $\omega$ is a bounded solution to (1.6) 
in $\left.\left\{\mathbb{R}^{3}-B(0, R)\right\} \times\right]-T_{1},+\infty[$ which vanishes for $t>0$. Then $\omega \equiv 0$ in $\left.\left\{\mathbb{R}^{3}-B(0, R)\right\} \times\right]-T_{1},+\infty[$.

The main point here is that we do not make any assumptions about $\omega$ on $\partial B(0, R)$. In fact, we can consider $\left.\omega\right|_{\partial B(0, R)}$ as a "control", and try to drive $\omega$ to zero by prescribing $\left.\omega\right|_{\partial B(0, R)}$. Our conjecture says that exact controllability is never possible in this case. Even the case $A=0$ and $B=0$ seems to be interesting, and we have not found it in the literature. One of our results in the paper is a proof of the conjecture when $A=0$ and $B=0$. We believe that the general case might be approachable by existing methods in the theory of unique continuation. By our results here, such a proof would give a solution to the regularity problem for the Navier-Stokes equations under condition (1.3).

\section{Notation and Main Results}

We denote by $\mathbb{M}^{3}$ the space of all real $3 \times 3$ matrices. Adopting summation over repeated Latin indices, running from 1 to 3, we shall use the following notation:

$$
\begin{gathered}
u \cdot v=u_{i} v_{i}, \quad|u|=\sqrt{u \cdot u}, \quad u=\left(u_{i}\right) \in \mathbb{R}^{3}, v=\left(v_{i}\right) \in \mathbb{R}^{3} ; \\
A: B=\operatorname{tr} A^{*} B=A_{i j} B_{i j}, \quad|A|=\sqrt{A: A}, \\
A^{*}=\left(A_{j i}\right), \quad \operatorname{tr} A=A_{i i}, \quad A=\left(A_{i j}\right) \in \mathbb{M}^{3}, B=\left(B_{i j}\right) \in \mathbb{M}^{3} ; \\
u \otimes v=\left(u_{i} v_{j}\right) \in \mathbb{M}^{3}, \quad A u=\left(A_{i j} u_{j}\right) \in \mathbb{R}^{3}, \quad u, v \in \mathbb{R}^{3}, A \in \mathbb{M}^{3} .
\end{gathered}
$$

Let $\omega$ be a domain in some finite-dimensional space. We denote by $L_{m}\left(\omega ; \mathbb{R}^{l}\right)$ and $W_{m}^{1}\left(\omega ; \mathbb{R}^{l}\right)$ the known Lebesgue and Sobolev spaces of functions from $\omega$ into $\mathbb{R}^{l}$. The norm of the space $L_{m}\left(\omega ; \mathbb{R}^{l}\right)$ is denoted by $\|\cdot\|_{m, \omega}$. If $m=2$, then we use the abbreviation $\|\cdot\|_{\omega} \equiv\|\cdot\|_{2, \omega}$.

Let $T$ and $T_{1}$ be two parameters such that $T_{1}<T, \Omega$ be a domain in $\mathbb{R}^{3}$. We denote by $\left.Q_{T_{1}, T} \equiv \Omega \times\right] T_{1}, T[$ the space-time cylinder. Space-time points are denoted by $z=(x, t), z_{0}=\left(x_{0}, t_{0}\right)$, etc. Let $L_{m, n}\left(Q_{T_{1}, T} ; \mathbb{R}^{l}\right)$ be the space 
of measurable $\mathbb{R}^{l}$-valued functions with the following norm

$$
\|f\|_{m, n, Q_{T_{1}, T}}= \begin{cases}\left(\int_{T_{1}}^{T}\|f(\cdot, t)\|_{m, \Omega}^{n} d t\right)^{\frac{1}{n}}, & n \in[1,+\infty[ \\ \operatorname{ess} \sup _{t \in] T_{1}, T[}\|f(\cdot, t)\|_{m, \Omega}, & n=+\infty .\end{cases}
$$

In the special case $\Omega=\mathbb{R}^{3}$ and $T_{1}=-T_{0}$ and $T=+\infty$, we abbreviate

$$
\begin{gathered}
L_{m}\left(\Omega ; \mathbb{R}^{3}\right)=L_{m}, \quad W_{2}^{1}\left(\Omega ; \mathbb{R}^{3}\right)=H^{1}, \quad L_{m, n}\left(Q_{T_{1}, T} ; \mathbb{R}^{3}\right)=L_{m, n}, \\
L_{m}\left(T_{1}, T ; W_{2}^{1}\left(\Omega ; \mathbb{R}^{3}\right)\right)=L_{m}\left(H^{1}\right) .
\end{gathered}
$$

For integrable in $Q_{T}$ scalar-valued, vector-valued, and tensor-valued functions, we shall use the following differential operators

$$
\begin{gathered}
\partial_{t} v=\frac{\partial v}{\partial t}, \quad v_{, i}=\frac{\partial v}{\partial x_{i}}, \quad \nabla p=\left(p_{, i}\right), \quad \nabla u=\left(u_{i, j}\right), \\
\operatorname{div} v=v_{i, i}, \quad \operatorname{div} \tau=\left(\tau_{i j, j}\right), \quad \Delta u=\operatorname{div} \nabla u,
\end{gathered}
$$

which are understood in the sense of distributions. Here $x_{i}, i=1,2,3$, are the Cartesian coordinates of a point $x \in \mathbb{R}^{3}$, and $\left.t \in\right] 0, T[$ is the time variable.

We recall to the reader the definition of the weak Leray-Hopf solution to the following Cauchy problem (see [4] and [8]):

$$
\begin{gathered}
\partial_{t} v(x, t)+\operatorname{div} v(x, t) \otimes v(x, t)-\Delta v(x, t)=-\nabla p(x, t), \\
\operatorname{div} v(x, t)=0
\end{gathered}
$$

for $x \in \mathbb{R}^{3}$ and for $t>-T_{0}$, and

$$
v\left(x,-T_{0}\right)=a(x), \quad x \in \mathbb{R}^{3} .
$$

Here, $T_{0}$ is a given positive parameter, and $a$ is a given divergence free function from $W_{2}^{1}\left(\mathbb{R}^{3} ; \mathbb{R}^{3}\right)$.

Definition 2.1 We say that a divergence free function

$$
v \in L_{2, \infty} \cap L_{2}\left(H^{1}\right)
$$


is called the weak Leray-Hopf solution to the Cauchy problem (2.1) and (2.2) if the following conditions holds:

$$
\begin{aligned}
& \text { for each } w \in L_{2} \text {, the function } t \mapsto \int_{\mathbb{R}^{3}} v(x, t) \cdot w(x) d x \text { is } \\
& \text { continuous at any point } t \in\left[-T_{0},+\infty[;\right. \\
& \int\left\{-v \cdot w_{t}-v \otimes v: \nabla w+\nabla v: \nabla w\right\} d z=0 \\
& \int_{-T_{0},+\infty}\left\{\begin{array}{l}
\text { for any divergence free function } w \in C_{0}^{\infty}\left(Q_{-T_{0},+\infty} ; \mathbb{R}^{3}\right) ;
\end{array}\right.
\end{aligned}
$$

for any $t \in\left[-T_{0},+\infty[\right.$, the following energy inequality is valid

$$
\begin{gathered}
\int_{\mathbb{R}^{3}}|v(x, t)|^{2} d x+2 \int_{-T_{0}}^{t} \int_{\mathbb{R}^{3}}|\nabla v|^{2} d x d t^{\prime} \leq \int_{\mathbb{R}^{3}}|a|^{2} d x \\
\|v(\cdot, t)-a(\cdot)\|_{L_{2}} \text { as } t \rightarrow+0 .
\end{gathered}
$$

One can show (see, for instance, [17]) that if, for a given weak solution, we introduce (normalized) pressure

$$
p(x, t) \equiv \frac{1}{4 \pi} \int_{\mathbb{R}^{3}} \frac{1}{|x-y|} \operatorname{div} \operatorname{div}(v(y, t) \otimes v(y, t)) d y,
$$

then the pair of $v$ and $p$ satisfies the Navier-Stokes equations in the sense of distribution.

In what follows, we always assume that

$$
v \in L_{3, \infty}
$$

This allows us to improve properties (2.3) and (2.5). Namely, instead of (2.3), we have now:

$$
\begin{gathered}
\text { for each } w \in L_{\frac{3}{2}} \text {, the function } t \mapsto \int_{\mathbb{R}^{3}} v(x, t) \cdot w(x) d x \text { is } \\
\text { continuous at any point } t \in\left[-T_{0},+\infty[.\right.
\end{gathered}
$$

Inequality (2.5) becomes

$$
\int_{\mathbb{R}^{3}}|v(x, t)|^{2} d x+2 \int_{-T_{0}}^{t} \int_{\mathbb{R}^{3}}|\nabla v|^{2} d x d t^{\prime}=\int_{\mathbb{R}^{3}}|a|^{2} d x
$$


for any $t \in\left[-T_{0},+\infty[\right.$. To see that, we note that

$$
v \in L_{4}
$$

and

$$
\operatorname{div}(v \otimes v) \in L_{\frac{4}{3}} \cap L_{\frac{6}{5}, 2},
$$

and, by the coercive $L_{s, l}$-estimates for solutions to the Cauchy problem for the linearized Navier-Stokes equations (see [3], [10] and [5], [7], and [20] in the case $s=l$ ),

$$
\left|\partial_{t} v\right|,\left|\nabla^{2} v\right|,|\nabla p| \in L_{\frac{4}{3}}\left(Q_{-T_{0}+\delta, T}\right) \cap L_{\frac{6}{5}, 2}\left(Q_{-T_{0}+\delta, T}\right)
$$

for any positive numbers $\delta$ and $T$ such that $-T_{0}+\delta<T$. Then, (2.10) easily follows from (2.6), (2.11), and (2.13). We also would like to note that (2.3) and (2.10) imply

$$
v \in C\left(\left[-T_{0}, T\right] ; L_{2}\right) .
$$

Another important consequence of assumption (2.8) is continuity of $v$ in time from the right with values in $L_{3}$, i.e.,

$$
\left\|v(\cdot, t)-v\left(\cdot, t_{0}\right)\right\|_{L_{3}} \rightarrow 0 \text { as } t \rightarrow t_{0} \text { and } t>t_{0} .
$$

In turn, according to $(2.7),(2.8)$, and (2.15), we see that

$$
p \in L_{\frac{3}{2}, \infty}
$$

and

$$
\left\|p(\cdot, t)-p\left(\cdot, t_{0}\right)\right\|_{L_{\frac{3}{2}}} \rightarrow 0 \text { as } t \rightarrow t_{0} \text { and } t>t_{0} .
$$

Given positive numbers $T_{1}$ and $R_{1}$, we let

$$
\left.\widetilde{Q}\left(T_{1}, R_{1}\right)=\left\{R^{3}-B\left(0, R_{1}\right)\right\} \times\right]-T_{1},+\infty[.
$$

Definition 2.2 Assume that $A=\left(A_{i}\right)$ and $B=\left(B_{i j}\right)$ are measurable and bounded functions on $\widetilde{Q}\left(T_{1}, R_{1}\right)$.

We say that the pair $(A, B)$ belongs to the class $\mathcal{C}\left(T_{1}, R_{1}\right)$ if the following condition holds. Whenever a function $\omega: \widetilde{Q}\left(T_{1}, R_{1}\right) \rightarrow \mathbb{R}^{3}$ satisfies

$$
\begin{gathered}
\text { (i) } \omega \text { and } \nabla \omega \text { are bounded and continuous, } \\
\lim _{|x| \rightarrow+\infty}|\omega(x, t)|=0 \text { uniformly in } t,
\end{gathered}
$$

(ii) $\partial_{t} \omega-\Delta \omega=A_{k} \omega_{, k}+B \omega$ in $\widetilde{Q}\left(T_{1}, R_{1}\right)$ (in the sense of distributions),

$$
\text { (iii) } \omega(x, t)=0, \quad x \in \mathbb{R}^{3}-B\left(0, R_{1}\right), t>0,
$$

then $\omega(x, t) \equiv 0$ in $\widetilde{Q}\left(T_{1}, R_{1}\right)$. 
Conjecture $H$ : Assume that, for $k=0,1, \ldots$, the functions $\nabla^{k} A$ and $\nabla^{k} B$ are Hölder continuous and bounded in $\widetilde{Q}\left(T_{1}, R_{1}\right)$,

$$
\lim _{R \rightarrow+\infty} \sup _{x \in \mathbb{R}^{3}-B(0, R)-T_{1}<t<+\infty} \sup _{-\nabla^{k} A(x, t)|+| \nabla^{k} B(x, t) \mid=0,}
$$

and

$$
\lim _{t \rightarrow 0} \sup _{x \in \mathbb{R}^{3}-B\left(0, R_{1}\right)}\left|\nabla^{k} A(x, t)\right|+\left|\nabla^{k} B(x, t)\right|=0 .
$$

Then $(A, B) \in \mathcal{C}\left(T_{1}, R_{1}\right)$.

Conjecture $G$ (Restricted Conjecture H) Assume that, for a solenoidal vector-valued function $w$, functions $A=-w$ and $B=\nabla w$ meet the conditions of Conjecture $\mathrm{H}$. Then $(A, B) \in \mathcal{C}\left(T_{1}, R_{1}\right)$.

Our main result is as follows.

Theorem 2.3 Suppose that Conjecture $G$ is true. Then, any weak LerayHopf solution to the Cauchy problem (2.1) and (2.2) is smooth.

We believe that Conjecture $\mathrm{H}$ is true although we have the proof of it only for the case $A=0$ and $B=0$ (for details, see section 4).

\section{Blow-up}

Assume that the statement of Theorem (2.3) is false. Without loss of generality, we may think that a singular point appears at time $t=0$ and is located at the origin.

Under our assumptions on $v$ and $p$, the pair $(v, p)$ is a suitable weak solution (for the definition, we refer the reader to [1]), i.e., it satisfies the local energy inequality. In our particular case, it satisfies even the local energy identity. Since $z=0$ is a singular point of our suitable weak solution, the theory of partial regularity for suitable weak solutions to the Navier-Stokes equations says (see [17]) that there exists a sequence of positive numbers $R_{k}$ such that $R_{k} \rightarrow 0$ as $k \rightarrow+\infty$ and

$$
A\left(R_{k}\right) \equiv \sup _{-R_{k}^{2} \leq t \leq 0} \frac{1}{R_{k}} \int_{B\left(0, R_{k}\right)}|v(x, t)|^{2} d x>\varepsilon_{\star}
$$

for all $k \in \mathbb{N}$. Here, $\varepsilon_{\star}$ is an absolute positive constant and $B(x, R)$ stands for the $3 \mathrm{D}$ ball of radius $R$ with the center at the point $x$. 
We extend functions $v$ and $p$ to the whole space $\mathbb{R}^{3+1}$ in the following way

$$
\begin{aligned}
& \tilde{v}(x, t)=\left\{\begin{array}{ccc}
v(x, t) & t \geq-T_{0} \\
0 & t<-T_{0} & x \in \mathbb{R}^{3}
\end{array}\right. \\
& \tilde{p}(x, t)=\left\{\begin{array}{ccc}
p(x, t) & t \geq-T_{0} \\
0 & t<-T_{0} & x \in \mathbb{R}^{3}
\end{array}\right.
\end{aligned}
$$

Now, we let

$$
v^{R_{k}}(x, t)=R_{k} \tilde{v}\left(R_{k} x, R_{k}^{2} t\right), \quad p^{R_{k}}(x, t)=R_{k} \tilde{p}\left(R_{k} x, R_{k}^{2} t\right)
$$

for $x \in \mathbb{R}^{3}$ and $t \in \mathbb{R}$. Obviously, for any $t \in \mathbb{R}$,

$$
\int_{\mathbb{R}^{3}}\left|v^{R_{k}}(x, t)\right|^{3} d x=\int_{\mathbb{R}^{3}}|\tilde{v}(x, t)|^{3} d x
$$

and

$$
\int_{\mathbb{R}^{3}}\left|p^{R_{k}}(x, t)\right|^{\frac{3}{2}} d x=\int_{\mathbb{R}^{3}}|\tilde{p}(x, t)|^{\frac{3}{2}} d x .
$$

Hence, without loss of generality, one may assume that

$$
v^{R_{k} \star} \rightarrow \quad \text { in } \quad L_{\infty}\left(\mathbb{R} ; L_{3}\right) \quad \text { as } \quad k \rightarrow+\infty,
$$

where $\operatorname{div} u=0$ in $\mathbb{R}^{3} \times \mathbb{R}$, and

$$
p^{R_{k} \stackrel{\star}{\rightarrow}} q \quad \text { in } \quad L_{\infty}\left(\mathbb{R} ; L_{\frac{3}{2}}\right) \quad \text { as } \quad k \rightarrow+\infty .
$$

To get more information about boundedness of various norms for functions $v^{R_{k}}$ and $p^{R_{k}}$, let us fix a cut-off function $\phi \in C_{0}^{\infty}\left(\mathbb{R}^{3+1}\right)$ and introduce the function $\phi^{R_{k}}$ in the following way

$$
\phi(y, \tau)=R_{k} \phi^{R_{k}}\left(R_{k} y, R_{k}^{2} \tau\right), \quad y \in \mathbb{R}^{3} \quad \tau \in \mathbb{R} .
$$

We choose $R_{k}$ so small to provide

$$
\operatorname{spt} \phi \subset\left\{(y, \tau) \| \tau>-\frac{T_{0}}{R_{k}}\right\} \Longrightarrow \operatorname{spt} \phi^{R_{k}} \subset\left\{(x, t) \| t>-T_{0}\right\} .
$$


Then, we have

$$
\begin{gathered}
2 \int_{-T_{0}}^{+\infty} \int_{\mathbb{R}^{3}} \phi^{R_{k}}|\nabla v|^{2} d z=\int_{-T_{0}}^{+\infty} \int_{\mathbb{R}^{3}}\left\{|v|^{2}\left(\Delta \phi^{R_{k}}+\partial_{t} \phi^{R_{k}}\right)\right. \\
\left.+v \cdot \nabla \phi^{R_{k}}\left(|v|^{2}+2 p\right)\right\} d z
\end{gathered}
$$

and after changing variables we arrived at the identity

$$
\begin{gathered}
2 \int_{\mathbb{R} \mathbb{R}^{3}} \phi\left|\nabla v^{R_{k}}\right|^{2} d z=\int_{\mathbb{R}} \int_{\mathbb{R}^{3}}\left\{\left|v^{R_{k}}\right|^{2}\left(\Delta \phi+\partial_{t} \phi\right)\right. \\
\left.+v^{R_{k}} \cdot \nabla \phi\left(\left|v^{R_{k}}\right|^{2}+2 p^{R_{k}}\right)\right\} d z .
\end{gathered}
$$

Now, from (3.2), (3.3), and (3.6), it follows that, for any domain $Q \Subset \mathbb{R}^{3+1}$,

$$
\int_{Q}\left|\nabla v^{R_{k}}\right|^{2} d z \leq c_{1}(Q)<+\infty .
$$

We emphasize that the constant in (3.7) is independent of $R_{k}$. Then, we apply known arguments, including multiplicative inequalities and $L_{s, l}$-coercive estimates for solutions to the non-stationary Stokes equations, and obtain the bound

$$
\int_{Q}\left(\left|v^{R_{k}}\right|^{4}+\left|\partial_{t} v^{R_{k}}\right|^{\frac{4}{3}}+\left|\nabla^{2} v^{R_{k}}\right|^{\frac{4}{3}}+\left|\nabla p^{R_{k}}\right|^{\frac{4}{3}}\right) d z \leq c_{2}(Q) .
$$

The latter together with (3.4) and (3.5) implies

$$
v^{R_{k}} \longrightarrow u \quad \text { in } \quad L_{3}\left(Q ; \mathbb{R}^{3}\right)
$$

for $Q \Subset \mathbb{R}^{3+1}$. Moreover, according to (3.4),(3.5), and (3.8), we see that, in addition,

$$
v^{R_{k}} \longrightarrow u \quad \text { in } \quad C\left([a, b] ; L_{2}\left(\Omega ; \mathbb{R}^{3}\right)\right)
$$

for any $-\infty<a<b<+\infty$ and for any $\Omega \in \mathbb{R}^{3}$.

Combining all information about limit functions $u$ and $q$ (see (3.2)-(3.10)), we obtain the followings facts:

$$
\int_{Q}\left(|u|^{4}+|\nabla u|^{2}+\left|\partial_{t} u\right|^{\frac{4}{3}}+\left|\nabla^{2} u\right|^{\frac{4}{3}}+|\nabla q|^{\frac{4}{3}}\right) d z \leq c_{3}(Q)
$$


for any $Q \Subset \mathbb{R}^{3+1}$;

$$
u \in C\left([a, b] ; L_{2}\left(\Omega ; \mathbb{R}^{3}\right)\right)
$$

for any $-\infty<a<b<+\infty$ and for any $\Omega \in \mathbb{R}^{3}$;

functions $u$ and $q$ satisfy the Navier-Stokes equations a.e. in $\mathbb{R}^{3}$;

$$
2 \int_{\mathbb{R}_{\mathbb{R}^{3}}} \phi|\nabla u|^{2} d z=\int_{\mathbb{R}_{\mathbb{R}^{3}}}\left\{|u|^{2}\left(\Delta \phi+\partial_{t} \phi\right)+u \cdot \nabla \phi\left(|u|^{2}+2 q\right)\right\} d z
$$

for all functions $\phi \in C_{0}^{\infty}\left(\mathbb{R}^{3+1}\right)$.

By (2.11)-(2.14), the pair $(u, q)$ is a suitable weak solution to the NavierStokes equation in $\mathbb{R}^{3+1}$.

Our next observation on limit functions comes from (2.15) and (2.17) for $t_{0}=0$. For any positive numbers $R$ and $t,(2.15)$ implies

$$
\int_{B(0, R)}\left|v^{R_{k}}(y, t)\right|^{3} d y=\int_{B\left(0, R_{k} R\right)}\left|v\left(x, R_{k}^{2} t\right)\right|^{3} d x \longrightarrow 0
$$

as $R_{k} \rightarrow 0$. This means that

$$
u(\cdot, t)=0, \quad t>0 .
$$

In the same way, we deduce from (2.17) that

$$
q(\cdot, t)=0, \quad t>0 .
$$

Finally, according to (3.1),

$$
\sup _{-R_{k}^{2} \leq t \leq 0} \frac{1}{R_{k}} \int_{B\left(0, R_{k}\right)}|v(x, t)|^{2} d x=\sup _{-1 \leq t \leq 0} \int_{B(0,1)}\left|v^{R_{k}}(x, t)\right|^{2} d x>\varepsilon_{\star}
$$

for all $k \in \mathbb{N}$, and, by (3.10), we obtain

$$
\sup _{-1 \leq t \leq 0} \int_{B(0,1)}|u(x, t)|^{2} d x>\varepsilon_{\star} .
$$

Proof of Theorem 2.3: First, we are going to show that there exits some positive numbers $R_{1}$ and $T_{1}$ such that, for any $k=0,1, \ldots$, the function $\nabla^{k} u$ is Hölder continuous and bounded on the set

$$
\left.\widetilde{Q}\left(2 T_{1}, R_{1} / 2\right)=\mathbb{R}^{3}-B\left(0, R_{1} / 2\right) \times\right]-2 T_{1},+\infty[.
$$


To this end, let us fix an arbitrary number $T_{1}>2$ and note that

$$
\int_{-4 T_{1}}^{+\infty} \int_{\mathbb{R}^{3}}\left(|u|^{3}+|q|^{\frac{3}{2}}\right) d z=\int_{-4 T_{1}}^{0} \int_{\mathbb{R}^{3}}\left(|u|^{3}+|q|^{\frac{3}{2}}\right) d z<+\infty .
$$

Therefore,

$$
\int_{-4 T_{1}}^{+\infty} \int_{\mathbb{R}^{3}-B(0, R)}\left(|u|^{3}+|q|^{\frac{3}{2}}\right) d z \rightarrow 0 \quad \text { as } R \rightarrow+\infty .
$$

This means that, for given $\varepsilon>0$, there exists a number $R_{1}\left(\varepsilon, T_{1}\right)>4$ such that

$$
\int_{-4 T_{1}}^{+\infty} \int_{\mathbb{R}^{3}-B\left(0, R_{1} / 24\right)}\left(|u|^{3}+|q|^{\frac{3}{2}}\right) d z<\varepsilon
$$

Now, assume that $z_{0}=\left(x_{0}, t_{0}\right) \in \widetilde{Q}\left(2 T_{1}, R_{1} / 2\right)$. Then,

$$
\left.Q\left(z_{0}, 1\right) \equiv B\left(x_{0}, 1\right) \times\right] t_{0}-1, t_{0}\left[\subset\left\{\mathbb{R}^{3}-B\left(0, R_{1} / 4\right)\right\} \times\right]-4 T_{1},+\infty[.
$$

So, by (3.18), one can claim that, for any $\varepsilon$,

$$
\int_{t_{0}-1}^{t_{0}} \int_{B\left(x_{0}, 1\right)}\left(|u|^{3}+|q|^{\frac{3}{2}}\right) d z<\varepsilon
$$

for $z_{0} \in \widetilde{Q}\left(2 T_{1}, R_{1} / 2\right)$, where $T_{1}>2$ and $R_{1}\left(\varepsilon, T_{1}\right)>4$. Then, it follows from (3.19), from the Caffarelli-Kohn-Nirenberg theorem, and from the regularity theory for solutions to the Stokes equations (for details see [1] and [13], Proposition 2.1) that, for each $k=0,1, \ldots$, there exists a number $c^{(k)}$ independent of $z_{0}$ such that

$$
\sup _{z \in Q\left(z_{0}, 1 / 2\right)}\left|\nabla^{k} u(z)\right| \leq c^{(k)}<+\infty .
$$

Hölder continuity of $\nabla^{k} u$ on $\widetilde{Q}\left(2 T_{1}, R_{1} / 2\right)$ is also a consequence of the regularity theory for the Stokes equations and bootstrap arguments. 
So, it remains to show that functions $A=-u$ and $B=\nabla u$ meet conditions (2.18) and (2.19). To see that let us introduce the sequence of functions

$$
v_{e}^{m}(x, t)=u(x+m e, t), \quad p_{e}^{m}(x, t)=q(x+m e, t)
$$

for $x \in B(0,2)$, for $e \in B(0,1)$, and for $-2 T_{1}<t<+\infty$. Obviously, for each fixed $m \in \mathbb{N}$ and for each fixed $e \in B(0,1)$, the pair $\left(v_{e}^{m}, p_{e}^{m}\right)$ is a suitable weak solution to the Navier-Stokes equations in

$$
B(0,2) \times]-2 T_{1},+\infty[.
$$

Moreover,

$$
\lim _{m \rightarrow+\infty} \int_{t_{0}-1}^{t_{0}} \int_{B(0,1)}\left(\left|v_{e}^{m}\right|^{3}+\left|p_{e}^{m}\right|^{\frac{3}{2}}\right) d x d t=0 .
$$

By the above arguments, one can claim that

$$
\left\|\nabla^{k} v_{e}^{m}\right\|_{C^{\alpha}\left(\bar{Q}\left(z_{0}, 1 / 2\right)\right)} \leq \widetilde{c}_{k}
$$

for some $\alpha \in] 0,1\left[\right.$. Here, $z_{0}=\left(0, t_{0}\right), C^{\alpha}(\bar{Q})$ is the space of functions which are continuous on the compact $\bar{Q}$ with respect to the usual parabolic distance. It is important to note constants $\widetilde{c}_{k}, k=0,1, \ldots$, are independent of $m, e$, and $t_{0}$. So, we see that, for $k=0,1, \ldots$,

$$
\sup _{e \in B(0,1)} \sup _{B(0,1 / 2)} \sup _{-T_{1} \leq t<+\infty}\left|\nabla^{k} v_{e}^{m}(x, t)\right| \rightarrow 0
$$

as $m \rightarrow+\infty$. This implies (2.18). Now, (2.19) follows from (2.18) and from Hölder continuity of $\nabla^{k} u$ on $\widetilde{Q}\left(2 T_{1}, R_{1} / 2\right)$.

Now, let $\omega$ be the vorticity of $u$, i.e.,

$$
\omega=\nabla \wedge u .
$$

The function $\omega$ meets the equation

$$
\left.\partial_{t} \omega+u_{k} \omega_{, k}-\omega_{k} u_{, k}-\Delta u=0, \quad \text { in } \mathbb{R}^{3} \times\right]-T_{1}, \infty[.
$$

By the conjecture,

$$
\omega(z)=0 \quad \text { if } z \in \widetilde{Q}\left(T_{1}, R_{1}\right)
$$


On the other hand, there exists an open subset $\mathcal{O} \subset]-T_{1}, 0[$ such that $|\mathcal{O}|=T_{1}$ and, for each $t \in \mathcal{O}$, the function $u$ is analytic in spatial variables. But then $\omega$ is also an analytic function in the same variables, and, therefore, (3.20) implies that

$$
\omega(\cdot, t)=0, \quad t \in \mathcal{O} .
$$

This means that, for each $t \in \mathcal{O}$, the function $u(\cdot, t)$ is harmonic and has the finite $L_{3}\left(\mathbb{R}^{3} ; \mathbb{R}^{3}\right)$-norm. It turn, this fact leads to the identity

$$
u(\cdot, t)=0, \quad t \in \mathcal{O}
$$

So, $u=0$ a. e. in $\left.\mathbb{R}^{3} \times\right]-T_{1}, \infty[$. This contradicts with (3.17). Theorem 2.3 is proved.

\section{A Backward Uniqueness Theorem for the Heat Equation}

In this section, we introduce additional notation:

$$
\begin{gathered}
\mathbb{R}_{+}^{n}=\left\{x \in \mathbb{R}^{n} \| x=\left(x_{1}, x_{2}, \ldots, x_{n}\right)=\left(x_{i}\right), x_{n}>0\right\}, \\
\left.Q_{T}=\mathbb{R}_{+}^{n} \times\right] 0, T[
\end{gathered}
$$

where $T$ is a positive fixed number.

Theorem 4.1 Let $u: Q_{T} \rightarrow \mathbb{R}$ be a bounded smooth function satisfying the heat equation $\partial_{t} u=\Delta u$ in $Q_{T}$. Assume that there exists a non-empty open set $\Omega \subset \mathbb{R}_{+}^{n}$ such that

$$
\lim _{t \rightarrow T-0} \int_{\Omega}|u(x, t)| d x=0 .
$$

Then $u \equiv 0$ in $Q_{T}$.

Proof. Using the known regularity theory for the heat equation and the fact that smooth solutions to the heat equation are analytic in spatial variables, we see that one can extend $u$ by zero to the set $\left.Q=\mathbb{R}_{+}^{n} \times\right] 0,+\infty[$, and the extension, also denoted by $u$, is smooth, satisfies the heat equation in $Q$, and vanishes for $t \geq T$. Also, replacing $u(x, t)$ by $u\left(x_{1}, x_{2}, \ldots, x_{n-1}, x_{n}+y_{n}, t+s\right)$ for small $y_{n}>0$ and $s>0$, we can assume that all derivatives of $u$ are 
well-defined, bounded and continuous in the closure $\bar{Q}$ of $Q$. Making these simplifying assumptions, we will now prove the theorem in several steps.

Step 1. Reduction to the case $n=1$. The obvious idea here is to use the Fourier transformation along $x^{\prime}=\left(x_{1}, x_{2}, \ldots, x_{n-1}\right)$. For each $t>0$ and $x_{n} \geq 0$, we define a distribution $\widetilde{u}\left(\cdot, x_{n}, t\right)$ on $\mathbb{R}^{n-1}$ by

$$
<\widetilde{u}\left(\cdot, x_{n}, t\right), \phi(\cdot)>=\int_{\mathbb{R}^{n-1}} d x^{\prime} u\left(x^{\prime}, x_{n}, t\right) \int_{\mathbb{R}^{n-1}} d \xi^{\prime} e^{t|\xi|^{2}-i x^{\prime} \cdot \xi^{\prime}} \phi\left(\xi^{\prime}\right) .
$$

Here, $\phi \in C_{0}^{\infty}\left(\mathbb{R}^{n-1}\right)$ and $\xi^{\prime}=\left(\xi_{1}, \xi_{2}, \ldots, \xi_{n-1}\right)$. Under suitable assumptions, $\widetilde{u}\left(\cdot, x_{n}, t\right)$ is a function and we have

$$
\widetilde{u}\left(\xi^{\prime}, x_{n}, t\right)=e^{t\left|\xi^{\prime}\right|^{2}} \int_{\mathbb{R}^{n-1}} u\left(x^{\prime}, x_{n}, t\right) e^{-i x^{\prime} \cdot \xi^{\prime}} d x^{\prime}
$$

A simple calculation shows that, for each fixed $\phi \in C_{0}^{\infty}\left(\mathbb{R}^{n-1}\right)$, the function

$$
\widetilde{u}_{\phi}\left(x_{n}, t\right)=<\widetilde{u}\left(\cdot, x_{n}, t\right), \phi(\cdot)>
$$

is bounded in $\left.\mathbb{R}_{+} \times\right] 0,+\infty\left[\right.$, satisfies the heat equation in $\left.\mathbb{R}_{+} \times\right] 0,+\infty[$, and vanishes for $t>T$. We now see that it is enough to prove the case $n=1$.

In what follows, we use the notation $\left.Q=\mathbb{R}_{+} \times\right] 0,+\infty[$, and $(\mathrm{x}, \mathrm{t})$ stands for points of $Q$.

Step 2. Reduction to the case $|u(x, t)| \leq C e^{-\alpha x}$. This can be achieved by the following change of variables:

$$
u(x, t)=v(x+2 \alpha t, t) e^{\alpha x+\alpha^{2} t}, \quad \alpha>0 .
$$

This function $v$ is, of course, defined in a domain different from $Q_{T}$, but we can obviously achieve by a suitable shift that the domain of $v$ contains a domain of the form $Q$ in which the theorem is violated, if $v$ does not identically vanish. Moreover, $v$ has the required decay as $x \rightarrow+\infty$.

Step 3. Proof in the case $n=1$ and $|u(x, t)| \leq C e^{-\alpha x}$. We extend $u$ to all $\mathbb{R} \times \mathbb{R}$ by requiring that the extension be an even function of $x$ vanishing for $t \in]-\infty, 0[\cup] T,+\infty[$. The extended function has a discontinuity in $t$ at $t=0$, but it is smooth in $\mathrm{t}$ (for a fixed $x$ ) when $t \in] 0,+\infty[$.

Let $a(x)=u(x, 0)$ and let

$$
g(t)=\lim _{x \rightarrow 0+0} 2 \frac{\partial u}{\partial x}(x, t) .
$$


Clearly,

$$
\frac{\partial u}{\partial t}(x, t)-\frac{\partial^{2} u}{\partial x^{2}}(x, t)=-\delta(x) g(t)+\delta(t) a(x), \quad x \in \mathbb{R}, \quad t \in \mathbb{R},
$$

where $\delta$ denotes the Dirac distribution.

Denoting by $\hat{g}$ and $\hat{a}$ the Fourier transformations of $g$ and $a$, respectively, we note that (4.1) implies

$$
\hat{g}\left(i \xi^{2}\right)=\hat{a}(\xi), \quad \xi \in \mathbb{R} .
$$

We now look at the function $\hat{g}$ in more detail. We have

$$
g(\tau)=\int_{\mathbb{R}} g(t) e^{-i \tau t} d t=\int_{0}^{T} g(t) e^{-i \tau t} d t .
$$

Hence, $\hat{g}$ is defined for each $\tau \in \mathbb{C}$ and is holomorphic in $\mathbb{C}$. Moreover, standard calculations together with (4.2) and the decay property of $a$ imply that

$$
\hat{g}(\tau)=\mathcal{O}\left(\frac{1}{|\tau|}\right) \quad \text { as } \tau \rightarrow \infty \text { in } K
$$

where $K=\{\tau \in \mathbb{C} \| \operatorname{Re} \tau=0$ or $\operatorname{Im} \tau=0\}$, i.e., $K$ is the union of the real and imaginary axes. In fact, (4.4) along the real axis and along the negative part of the imaginary axis follows from (4.3) and integration by parts. (4.4) along the positive part of imaginary axis follows from (4.2) and the fact that

$$
a(\xi)=\mathcal{O}\left(\frac{1}{|\xi|^{2}}\right) \quad \text { as } \xi \rightarrow \infty \text { and } \xi \in \mathbb{R} .
$$

Step 4. The last step in the proof is simple lemma about holomorphic functions.

Lemma 4.2 Let $K \subset \mathbb{C}$ be the union of the real and imaginary axes. Let $f: \mathbb{C} \rightarrow \mathbb{C}$ be a holomorphic function satisfying two conditions:

$$
|f(z)| \leq A e^{a|z|}, \quad z \in \mathbb{C},
$$

for some positive constants $a$ and $A$, and

$$
f(\tau)=\mathcal{O}\left(\frac{1}{|\tau|}\right) \quad \text { as } \tau \rightarrow \infty \text { in } K .
$$

Then $f \equiv 0$.

We leave proof of the lemma to the reader as an interesting exercise. We note that Lemma 4.2 can be easily obtained from the Phragmén-Lindelöf theorem for an angle (for details, see [11], Theorem 7.5). Theorem 4.1 is proved. 


\section{References}

[1] Caffarelli, L., Kohn, R.-V., Nirenberg, L., Partial regularity of suitable weak solutions of the Navier-Stokes equations, Comm. Pure Appl. Math., Vol. XXXV (1982), pp. 771-831.

[2] Foias, C., Une remarque sur l'unicité des solutions des equations de Navier-Stokes en dimension n, Bull. Soc. Math. France, 89(1961), pp. $1-8$.

[3] Giga, Y., Sohr, H., Abstract $L^{p}$-estimates for the Cauchy problem with applications to the Navier-Stokes equations in exterior domains, J. Funct. Anal. 102 (1991), 72-94.

[4] Hopf, E., Über die Anfangswertaufgabe für die hydrodynamischen Grundgleichungen, Math. Nachrichten, 4 (1950-51), 213-231.

[5] Ladyzhenskaya, O. A., Mathematical problems of the dynamics of viscous incompressible fluids, Fizmatgiz, Moscow 1961; English transltion, Gordon and Breach, New York-London, 1969.

[6] Ladyzhenskaya, O. A., On uniqueness and smoothness of generalized solutions to the Navier-Stokes equations, Zapiski Nauchn. Seminar. POMI, 5(1967), pp. 169-185.

[7] Ladyzhenskaya, O. A., Mathematical problems of the dynamics of viscous incompressible fluids, 2nd edition, Nauka, Moscow 1970.

[8] Leray, J., Sur le mouvement d'un liquide visqueux emplissant l'espace, Acta Math. 63(1934), pp. 193-248.

[9] Kozono, H., Sohr, H., Remarks om uniqueness of weak solutions to the Navier-Stokes equations, Analysis 16(1996), pp. 255-271.

[10] Maremonti, P., Solonnikov, V. A., On the estimate of solutions of evolution Stokes problem in anisotropic Sobolev spaces with a mixed norm, Zap. Nauchn. Sem. LOMI, 223(1994), 124-150.

[11] Markushevich, A. I., Theory of functions of a complex variable, Chelsea Pubishing Company, New York, 1977. 
[12] Masuda, K., Weak solutions of the Navier-Stokes equations, Tohoku Math. J., 36(1984), pp. 623-646

[13] Nečas, J., Ruzička, M., Šverák, V., On Leray's self-similar solutions of the Navier-Stokes equations, Acta Math., 176(1996), pp. 283-294.

[14] Neustupa, J., Partial regularity of weak solutions to the Navier-Stokes equations in the class $L^{\infty}\left(0, T ; L^{3}(\Omega)^{3}\right)$, J. math. fluid mech., 1(1999), pp. 309-325.

[15] Prodi, G., Un teorema di unicità per el equazioni di Navier-Stokes, Ann. Mat. Pura Appl., 48(1959), pp. 173-182.

[16] Seregin, G. A. On the number of singular points of weak solutions to the Navier-Stokes equations, Comm. Pure Appl. Math., 54(2001), issue 8, pp. 1019-1028.

[17] Seregin, G.A., Šverák, V., On solutions to the Navier-Stokes equations with lower bounds on pressure, to appear in Arch. Rational Mech. Anal.

[18] Serrin, J., The initial value problem for the Navier-Stokes equations. Nonlinear Problems (R. Langer ed.), pp. 69-98, Madison: The University of Wisconsin press, 1963.

[19] Sohr, H., v. Wahl, W., On the singular set and the uniqueness of weak solutions of the Navier-Stokes equations, manuscripta math.,

[20] Solonnikov, V. A., Estimates of solutions to the linearized system of the Navier-Stokes equations, Trudy of the Steklov Math. Inst., Vol. LXX, 1964, pp. 213-317.

G. Seregin

Steklov Institute of Mathematics at St.Petersburg,

St.Peterburg, Russia

V. Šverák

School of Mathematics, University of Minnesota, Minneapolis, USA 